\title{
Paternal support and preterm birth, and the moderation of effects of chronic stress: a study in Los Angeles County mothers
}

\author{
Jo Kay C. Ghosh • Michelle H. Wilhelm • \\ Christine Dunkel-Schetter • Christina A. Lombardi • \\ Beate R. Ritz
}

Received: 28 October 2009 / Accepted: 30 November 2009 / Published online: 12 January 2010

(C) The Author(s) 2009. This article is published with open access at Springerlink.com

\begin{abstract}
Maternal psychosocial stress is an important risk factor for preterm birth, but support interventions have largely been unsuccessful. The objective of this study is to assess how support during pregnancy influences preterm birth risk and possibly ameliorates the effects of chronic stress, life event stress, or pregnancy anxiety in pregnant
\end{abstract}

J. K. C. Ghosh $(\bowtie) \cdot$ M. H. Wilhelm • C. A. Lombardi •

B. R. Ritz

Department of Epidemiology, University of California,

Los Angeles,

650 Charles E. Young Dr. South, CHS 71-254,

Los Angeles, CA 90095-1772, USA

e-mail: jokay@ucla.edu

M. H. Wilhelm

e-mail: mwilhelm@ucla.edu

B. R. Ritz

e-mail: britz@ucla.edu

M. H. Wilhelm • B. R. Ritz

Center for Occupational and Environmental Health,

University of California, Los Angeles,

650 Charles E. Young Dr. South, CHS 71-254,

Los Angeles, CA 90095-1772, USA

C. Dunkel-Schetter

Department of Psychology, University of California, Los Angeles,

1285A Franz Hall, 405 Hilgard Ave,

Los Angeles, CA 90095-1563, USA

e-mail: dunkel@psych.ucla.edu

C. A. Lombardi

Center for Health Policy Research, University of California,

Los Angeles,

10960 Wilshire Blvd, Suite 1550,

Los Angeles, CA 90024, USA

e-mail: clombard@ucla.edu women. We examined 1,027 singleton preterm births and 1,282 full-term normal weight controls from a populationbased retrospective case-control study of Los Angeles County, California women giving birth in 2003, a mostly Latina population (both US-born and immigrant). We used logistic regression to assess whether support from the baby's father during pregnancy influences birth outcomes and effects of chronic stress, pregnancy anxiety, and life event stress. Adjusted odds of preterm birth decreased with better support (OR 0.73 [95\%CI 0.52, 1.01]). Chronic stress (OR 1.46 [95\%CI 1.11, 1.92]), low confidence of a normal birth (OR 1.57 [95\% CI 1.17, 2.12]), and fearing for the baby's health (OR 1.67 [95\%CI 1.30, 2.14]) increased preterm birth risk, but life events showed no association. Our data also suggested that paternal support may modify the effect of chronic stress on the risk of preterm birth, such that among mothers lacking support, those with moderateto-high stress were at increased odds of delivering preterm (OR 2.15 [95\%CI 0.92, 5.03]), but women with greater support had no increased risk with moderate-to-high chronic stress (OR 1.13 [95\%CI 0.94, 1.35]). Paternal support may moderate the effects of chronic stress on the risk of preterm delivery.

Keywords Latina health · Pregnancy · Preterm birth . Social support $\cdot$ Psychosocial stress

\section{Introduction}

As the leading cause of infant mortality and a significant contributor to long-term health issues, preterm birth persists as a major public health problem in the United States and internationally. The prevalence of singleton preterm births 
in the U.S. is currently around $12.5 \%$, an $18 \%$ increase since 1990 (Martin et al. 2006). Despite many known risk factors, prevention efforts have not succeeded in reducing the prevalence of preterm birth (Committee on Understanding Premature Birth and Assuring Healthy Outcomes 2007).

Studies of maternal psychosocial stress have mostly shown positive associations with preterm birth (Badr et al. 2005; Hedegaard et al. 1996; Hobel et al. 2008; Hoffman and Hatch 1996; Rini et al. 1999; Rondo et al. 2003). Chronic stress, pregnancy-specific anxiety, stressful life events, and physical stress have all been associated with increased risk (Dole et al. 2003, 2004; Hobel and Culhane 2003; Hogue et al. 2001), with relatively consistent positive associations for chronic stress (Copper et al. 1996; Dole et al. 2003; Hedegaard et al. 1993; Hobel et al. 2008; Nordentoft et al. 1996; Paarlberg et al. 1995) and pregnancy-specific anxiety (Rini et al. 1999; Roesch et al. 2004; Wadhwa et al. 1993). The literature describes several plausible biological mechanisms, including activation of the neuroendocrine system, increasing susceptibility to infections, and stimulation of inflammatory processes that can trigger early parturition (Dudley 1999; Hobel 2004; Kramer et al. 2001; Wadhwa et al. 2001). Maternal stress results in increased cortisol and corticotrophin-releasing hormone (CRH), some of which passes into the fetal system (Dudley 1999; Hobel 2004; Sandman et al. 2006; Wadhwa et al. 2001, 2004; Weinstock 2005). Elevated CRH levels may initiate premature labor, reduce placental perfusion, and/or increase the release of cortisol (Knackstedt et al. 2005; McLean and Smith 2001; Ruiz et al. 2002; Sandman et al. 2006). Chronic stress also can stimulate a pro-inflammatory immune response and impaired growth hormone secretion (Dudley 1999; Knackstedt et al. 2005; Wadhwa et al. 2001). Beyond its direct physiological effects, stress may also lead to poor health behaviors, such as smoking, lack of physical exercise, and poor diet (Kramer et al. 2001).

Although a few studies have suggested that social support from a woman's network of family and friends, her neighborhood environment, and her intimate partner mitigate negative effects of stress during pregnancy (Collins et al. 1993; Dole et al. 2003; Elsenbruch et al. 2007; Feldman et al. 2000; Rothberg and Lits 1991), the majority of studies have not shown that such support reduces the risks of preterm birth (Bryce et al. 1988; Hedegaard et al. 1996; Villar et al. 1992). All of these studies were clinic-based rather than population-based, and many had limited statistical power due to small sample sizes or low prevalence of preterm birth. Additionally, most studies focused on only one or two measures of stress, making it difficult to draw conclusions about the effects of different types of stress. Here, we present the results from a population-based case-control study of 2,309 women who resided in Los Angeles (LA) County, California and gave birth in 2003. In a structured questionnaire, we ascertained information retrospectively about chronic stress, pregnancy anxiety, stressful life events, and paternal support during pregnancy. We examined associations between these measures of stress and preterm birth for the population as a whole and for immigrant and US-born Latinas in particular. In the first large-scale epidemiologic study of support in pregnancy, we explored the potential protective influences of paternal support directly on outcomes and as a modifier or buffer of stress effects.

\section{Methods}

\section{Subject selection}

The UCLA Environment and Pregnancy Outcomes Study (EPOS) is a case-control study nested within the year 2003 birth cohort of all women who resided in select zip codes of LA County (Ritz et al. 2007). The primary goal of EPOS was to study the effects of ambient air pollution on adverse birth outcomes. We used California State and LA County electronic birth certificate records to select live singleton births to mothers residing in zip codes near air pollution monitoring stations and/or major roadways. We included all cases of preterm birth $(<37$ weeks completed gestation) and low birthweight $(<2,500 \mathrm{~g})$ from zip codes located near air monitoring stations, and a random sample of $30 \%$ of cases from the remainder of the selected zip codes. Controls (fullterm, normal weight babies) were matched to cases based on birth month, month that data were received from the county health department, and zip code set, for a total sample of 6,374 women. We reached and enrolled 2,543 of these women $(40 \%$ response rate) approximately $3-$ 6 months after delivery. Mothers were interviewed in English or Spanish by telephone, by mail survey, and during home visits. Information about maternal age, race/ ethnicity, education, birthplace, parity, baby's sex, prenatal care payment source, and complications of pregnancy ${ }^{1}$ and delivery was obtained from birth certificates. The EPOS survey questionnaire provided detailed information on other risk factors such as smoking, alcohol consumption, and psychosocial factors. We used multiple imputation software (Raghunathan et al. 2002) to impute missing data on family income and maternal education based on individual and census block group characteristics. This work was approved

\footnotetext{
${ }^{1}$ Pregnancy complications defined as having one or more of the following: preeclampsia, eclampsia, chronic hypertension, renal disease, pyelonephritis, cardiac disease, acute or chronic lung disease, RH sensitization, hemoglobinopathy, uterine bleeding before labor, polyhydraminios/oligohydraminios, incompetent cervix, premature labor, sexually transmitted diseases other than genital herpes, hepatitis $\mathrm{B}$, rubella, and tocolysis
} 
by the UCLA Office for the Protection of Human Subjects and the California Committee for the Protection of Human Subjects.

To examine the potential for selection bias, we compared the distributions of demographic factors reported on birth certificates for survey responders versus non-responders, and found that the distributions were similar for the risk factors considered, except there were slightly more nonHispanic whites, educated mothers, and US-born women among the responders (Ritz et al. 2007). For the analyses presented here, we excluded one woman who identified herself as widowed because paternal support during pregnancy in this case was not defined, leaving for analysis 1,027 preterm births and 1,282 full-term normal weight controls (total $N=2,309$ ).

\section{Stress and support assessment}

We collected information on many known risk factors for preterm birth, and on the physical and psychosocial environment during pregnancy. Because the focus of the survey was environmental exposures, we used only a subset of items from each stress and support scale to reduce the burden on respondents. Paternal support was assessed using three questions from an instrument developed by one of the authors, and used in previous studies of pregnancy outcomes in LA County (Collins et al. 1993; Feldman et al. 2000; Turner et al. 1990). Specifically, we asked how often the baby's father showed he cared about her, criticized her, and supported her financially while she was pregnant. Response options were provided as a five-point Likert scale (never, almost never, sometimes, often, almost always), and we summed across all items to generate a cumulative total support score. Scores ranging from 3-8 were categorized as "Low" paternal support, while scores of 9-15 were "Moderate to High" paternal support (i.e., an average response of "sometimes" or more frequent support for each question). Moderate support (scores 9-11) was combined with High support (scores 12+) because effect estimates were similar for these groups.

The measures of stress during pregnancy employed here were also used previously by one of the authors and have been validated in English and Spanish in birth outcomes research (Dominguez et al. 2008; Rini et al. 1999; Roesch et al. 2004; Wadhwa et al. 1993). For each of the three stress measures, we selected a subset of the original items for brevity based on analyses of other pregnancy data sets indicating that these items were most predictive of adverse birth outcomes (Rini et al. 1999). The survey included two items on pregnancy anxiety (fearfulness for the baby's health, and lack of confidence of a normal birth; Rini et al. 1999), and four questions from the Perceived Stress Scale (PSS), a validated instrument measuring perception of stress (Cohen et al. 1983); which is referred to here as "chronic stress". We asked how often the woman felt: (1) she was able to control the important things in her life, (2) had difficulties piling up so high she felt she could not overcome them, (3) she was confident about her ability to handle her personal problems, and (4) things were "going her way" during her pregnancy. Chronic stress responses were recorded on a five-point Likert scale (never, almost never, sometimes, fairly often, very often) and summed to create a score (4-8 low, 9-12 moderate, 13-20 high stress). We also asked whether six major stressful life events occurred during pregnancy (losing a home or job, major conflicts with partner, having a close person experience substance abuse, alcohol, medical or legal problems, having a close person die, being threatened with physical harm, and having felt discrimination based on race/ethnicity). Life events responses were summed and we counted one event as "moderate life event stress", and two or more events as "high life event stress".

\section{Statistical methods}

We examined the relations between paternal support, stress, pregnancy anxiety, and preterm birth using crude and adjusted logistic regression models. We tested the influence of some well-known risk factors, including maternal age $(<20,20-24,25-29,30-34,35+$ years), race/ethnicity (NonHispanic White, Hispanic/Latina, Black, Asian/Other), education $(\leq 8,9-11,12,13-15,16+$ years $)$, parity $(0, \geq 1)$, annual household income $(<\$ 40,000 /$ year, $\geq \$ 40,000 /$ year $)$, marital status (married, living with partner, single/separated/ divorced), birthplace (US-born, foreign-born), smoking status (pregnancy smoker, former smoker, never smoker), alcohol use during pregnancy (yes, no), timing of prenatal care (beginning in first trimester, or after first trimester/no prenatal care), and payment source for prenatal care as a proxy indicator for socioeconomic status (private insurance or self pay vs. other insurance or no insurance) on relations between support, stress and preterm birth (Table 1). Additional confounders considered include the baby's sex, whether or not the mother worked outside the home, physical job stress based on job title, last live birth within the past year, having a previous preterm birth, pre-pregnancy BMI, living with one or more smokers, urogenital infections, and relevant pregnancy complications.

Adjustment for factors we included in our final modelsmaternal age, race/ethnicity, and marital status - changed the main support effect estimates by approximately $5 \%$ in our model of support and chronic stress. Other risk factors did not change the effect estimates for support by more than $3 \%$ or had missing data that we could not reasonably impute (e.g., pre-pregnancy weight), and were therefore left out of the final models. Addition of the final risk factors or any 
Table 1 Number (percent) of subjects in each outcome group by demographic characteristics and crude odds ratios [95\% CI]

$\begin{array}{llll}\text { Parameter } & \begin{array}{l}\text { Preterm birth }(N=1,027) \\ n(\text { column \%) }\end{array} & \begin{array}{l}\text { Term normal weight }(N=1,282) \\ n \text { (column \%) }\end{array} & \text { Preterm crude OR [95\% CI] }\end{array}$

\section{Maternal age (years)}

$<20$

20-24 (reference)

25-29

30-34

$35+$

Maternal race/ethnicity

Non-Hispanic White (reference)

Latina

Black

Asian $^{\mathrm{a}}$ or other ${ }^{\mathrm{b}}$

Missing

Maternal education (years)

$\leq 8$

9-11

12 (reference)

13-15

$16+$

Missing

Parity

$\geq 1$

0 (reference)

Household income

$<\$ 40,000 /$ year

$\geq \$ 40,000 /$ year (reference)

Missing

Mother's marital status

Married (reference)

Living with partner

Single, divorced, separated

Missing

Mother's birthplace

US-born (reference)

Foreign-born

Missing

Smoking status

Non-smoker (reference)

Former smoker

Pregnancy smoker

Missing
635 (61.8)

$392(38.2)$

635 (61.8)

$224(21.8)$

168 (16.4)

534 (52.3)

272 (26.6)

215 (20.9)

$6(0.6)$

$416(40.5)$

609 (59.3)

$2(0.2)$

$120(9.4)$

268 (20.9)

$373(29.1)$

338 (26.4)

183 (14.3)

$252(19.7)$

$842(65.7)$

$73(5.7)$

$107(8.4)$

$8(0.6)$

161 (12.6)

$266(20.8)$

332 (25.9)

$192(15.0)$

185 (18.0)

14 (1.4)

$769(60.0)$

$513(40.0)$

$746(58.2)$

338 (26.4)

198 (15.4)

755 (59.2)

266 (20.9)

254 (19.8)

$7(0.6)$

534 (41.7)

748 (58.4)

$0(0.0)$

726 (70.7)

243 (23.7)

57 (5.6)

$1(0.1)$

875 (68.3)

349 (27.2)

56 (4.4)

$2(0.2)$
$1.21[0.89,1.65]$

1.0

0.78 [0.61, 0.99]

$0.93[0.73,1.18]$

$1.23[0.94,1.62]$

$(p<0.01)$

1.0

$1.43[1.14,1.79]$

$2.02[1.39,2.92]$

$1.19[0.83,1.70]$

$(p<0.01)$

$0.99[0.75,1.30]$

$0.94[0.74,1.19]$

1.0

$0.94[0.72,1.22]$

$0.67[0.53,0.85]$

$(p=0.01)$

$1.08[0.92,1.28]$

1.0

$(p=0.37)$

$1.29[1.05,1.57]$

1.0

$(p=0.01)$

1.0

$1.45[1.18,1.77]$

$1.20[0.97,1.48]$

$(p<0.01)$

1.0

$1.05[0.88,1.24]$

$(p=0.60)$

1.0
$0.84[0.69,1.01]$

$1.23[0.84,1.79]$

$(p=0.09)$ 
Table 1 (continued)

\begin{tabular}{|c|c|c|c|}
\hline Parameter & $\begin{array}{l}\text { Preterm birth }(N=1,027) \\
n(\text { column \%) }\end{array}$ & $\begin{array}{l}\text { Term normal weight }(N=1,282) \\
n(\text { column } \%)\end{array}$ & Preterm crude OR $[95 \% \mathrm{CI}]$ \\
\hline \multicolumn{4}{|l|}{ Alcohol use during pregnancy } \\
\hline Yes & $90(8.8)$ & $111(8.7)$ & $1.02[0.76,1.36]$ \\
\hline No (reference) & $935(91.0)$ & $1170(91.3)$ & 1.0 \\
\hline \multirow[t]{2}{*}{ Missing } & $2(0.2)$ & $1(0.1)$ & \\
\hline & & & $(p=0.92)$ \\
\hline \multicolumn{4}{|l|}{ Prenatal care } \\
\hline Began in 1st trimester (reference) & $915(89.1)$ & $1196(93.3)$ & 1.0 \\
\hline Began after 1 st trimester, or no prenatal care & $103(10.1)$ & $79(6.2)$ & $1.70[1.26,2.31]$ \\
\hline Missing & $9(0.9)$ & $7(0.6)$ & $(p<0.01)$ \\
\hline \multicolumn{4}{|l|}{ Primary source of payment for prenatal care } \\
\hline Private insurance or self pay (reference) & $394(38.4)$ & $557(43.5)$ & 1.0 \\
\hline Other insurance or no insurance ${ }^{c}$ & $632(61.5)$ & $722(56.3)$ & $1.24[1.05,1.46]$ \\
\hline \multirow[t]{2}{*}{ Missing } & $1(0.1)$ & $3(0.20)$ & \\
\hline & & & $(p=0.01)$ \\
\hline
\end{tabular}

${ }^{a}$ Includes Chinese, Japanese, Korean, Vietnamese, Cambodian, Thai, Laotian, Filipino, Indian, other Asian

${ }^{\mathrm{b}}$ Includes Native American, Eskimo, Aleut, Hawaiian, Guamanian, Samoan, Pacific Islanders, and others

${ }^{\mathrm{c}}$ Includes Medicaid, Medicare, Worker's compensation, Title V, No charge, Medically indigent, Medi-Cal CPS Program, no prenatal care, and other government and non-government programs

other risk factors considered did not change the effect estimates and confidence intervals for the stress measures substantially.

\section{Results}

Nearly $80 \%$ of the women in our study were married $(55.8 \%)$ or living with a partner $(23.3 \%)$ during their pregnancy, and more than $50 \%$ were born outside the US, mostly in Mexico (Table 1). Over 65\% identified as Hispanic/Latino, $17 \%$ as Non-Hispanic White, $7 \%$ as Black, and the remainder of the women were Asian (including Chinese, Japanese, Korean, Vietnamese, Cambodian, Thai, Laotian, Filipino, Indian, other Asian) or Other (Native American and others). Higher odds of preterm birth were seen among Blacks and Latinas, in the youngest and oldest age groups, unmarried women, and women from low-income families. Few women reported smoking or drinking alcohol during pregnancy, and most received prenatal care beginning in the first trimester. Smoking and late start to prenatal care also showed positive associations with preterm birth, but alcohol use showed no association in crude models, likely due to low frequency.

Only $8 \%$ of women had low support scores, and $37 \%$ and 14\% reported moderate and high levels of chronic stress, respectively. Twenty-nine percent reported having experienced one of the six stressful life events during pregnancy, and $23 \%$ reported two or more life events (remainder experienced none). Commonly reported life events included having a serious argument with her partner (30.4\%) and having a close person experience substance abuse, medical, or legal trouble (19.0\%).

Women reporting low support and/or high levels of chronic stress were more likely to be young, less educated, Black or US-born Latina, single, separated, or divorced, and of low SES (based on household income or prenatal care payment source; Table 2). These women were also more likely to report life events during pregnancy. Black mothers reported the most chronic stress $(21.6 \%$ high stress), as well as having experienced more stressful life events, including experiencing racial discrimination during pregnancy more often than any of the other race groups $(5.7 \%)$. Women aged 35 or older and those with lower household incomes were also less confident about having a normal birth and more fearful for the health of the baby (results not shown).

Among Latina mothers in our study, 71\% were foreignborn, mostly in Mexico. However, US-born Latinas reported more life events during pregnancy (29.6\% with $2+$ events, compared to $19.1 \%$ of foreign-borns) and somewhat higher chronic stress $(19.1 \%$ vs. $14.9 \%$ of foreign-borns; Table 3). US-born Latina mothers were also more than twice as likely to report having low support $(14.3 \%)$ compared to foreign-borns (6.2\%). However, similar high levels of support were reported among those 
Table 2 Distributions of paternal support, chronic stress (Perceived Stress Scale), and life event stress by major demographic characteristics

\begin{tabular}{|c|c|c|c|c|c|c|c|c|}
\hline & $\begin{array}{l}\text { Low } \\
\text { paternal } \\
\text { support } \\
n \text { (row \%) }\end{array}$ & $\begin{array}{l}\text { Moderate-to- } \\
\text { high paternal } \\
\text { support } \\
n \text { (row \%) }\end{array}$ & $\begin{array}{l}\text { Low } \\
\text { chronic } \\
\text { stress } \\
n(\text { row \%) }\end{array}$ & $\begin{array}{l}\text { Moderate } \\
\text { chronic stress } \\
n(\text { row \%) }\end{array}$ & $\begin{array}{l}\text { High } \\
\text { chronic } \\
\text { stress } \\
n(\text { row \%) }\end{array}$ & $\begin{array}{l}\text { No life } \\
\text { events } \\
n \text { (row \%) }\end{array}$ & $\begin{array}{l}1 \text { life } \\
\text { event } \\
n \text { (row \%) }\end{array}$ & $\begin{array}{l}2+\text { life } \\
\text { events } \\
n \text { (row \%) }\end{array}$ \\
\hline \multicolumn{9}{|l|}{ Maternal age (years) } \\
\hline$<20$ & $35(15.5)$ & $191(84.5)$ & $90(39.7)$ & $89(39.2)$ & $48(21.2)$ & $104(45.2)$ & $67(29.1)$ & $59(25.7)$ \\
\hline $20-24$ & $41(8.5)$ & $439(91.5)$ & $182(38.5)$ & $205(43.3)$ & $86(18.2)$ & $208(43.6)$ & $138(28.9)$ & $131(27.5)$ \\
\hline $25-29$ & $41(6.9)$ & $557(93.1)$ & $291(48.7)$ & $202(33.8)$ & $104(17.4)$ & $278(46.4)$ & $187(31.2)$ & $134(22.4)$ \\
\hline $30-34$ & $28(4.8)$ & $555(95.2)$ & $316(54.4)$ & $210(36.1)$ & $55(9.5)$ & $309(52.8)$ & $169(28.9)$ & $107(18.3)$ \\
\hline $35+$ & $33(9.2)$ & $325(90.8)$ & $211(58.8)$ & $119(33.2)$ & $29(8.1)$ & $190(52.3)$ & $97(26.7)$ & $76(20.9)$ \\
\hline \multicolumn{9}{|l|}{ Maternal race/ethnicity } \\
\hline Non-Hispanic White & $18(4.6)$ & $374(95.4)$ & $262(66.2)$ & $104(26.3)$ & $30(7.6)$ & $209(52.6)$ & $108(27.2)$ & $80(20.2)$ \\
\hline Latina & $130(8.6)$ & 1384 (91.4) & $668(44.4)$ & $593(39.5)$ & $242(16.1)$ & $744(49.1)$ & $435(28.7)$ & $336(22.2)$ \\
\hline Black & $21(13.9)$ & $130(86.1)$ & $65(42.5)$ & $55(36.0)$ & $33(21.6)$ & $39(24.8)$ & $59(37.6)$ & $59(37.6)$ \\
\hline Asian $^{\mathrm{a}}$ or other ${ }^{\mathrm{b}}$ & $9(5.1)$ & $166(94.9)$ & $91(52.9)$ & $65(37.8)$ & $16(9.3)$ & $88(50.9)$ & $55(31.8)$ & $30(17.3)$ \\
\hline \multicolumn{9}{|c|}{ Maternal education (years) } \\
\hline$\leq 8$ & $16(5.4)$ & $278(94.6)$ & $113(38.8)$ & $135(46.4)$ & $43(14.8)$ & $184(61.7)$ & $69(23.2)$ & $45(15.1)$ \\
\hline $9-11$ & $57(12.2)$ & $412(87.9)$ & $185(39.4)$ & $196(41.7)$ & 89 (18.9) & $232(49.0)$ & $141(29.8)$ & $101(21.3)$ \\
\hline 12 & $58(9.4)$ & $557(90.6)$ & $270(44.1)$ & $245(40.0)$ & $98(16.0)$ & $250(40.9)$ & $197(32.2)$ & $165(27.0)$ \\
\hline $13-15$ & $28(8.1)$ & $320(92.0)$ & $184(53.3)$ & $104(30.1)$ & $57(16.5)$ & $146(41.7)$ & $106(30.3)$ & $98(28.0)$ \\
\hline $16+$ & $17(3.5)$ & $465(96.5)$ & $321(66.5)$ & $132(27.3)$ & $30(6.2)$ & $261(53.7)$ & $132(27.2)$ & $93(19.1)$ \\
\hline \multicolumn{9}{|l|}{ Parity } \\
\hline$\geq 1$ & $107(7.8)$ & $1263(92.2)$ & $626(45.9)$ & $537(39.4)$ & $201(14.7)$ & $654(47.8)$ & $402(29.4)$ & $312(22.8)$ \\
\hline 0 & $71(8.1)$ & $804(91.9)$ & $464(53.2)$ & $288(33.0)$ & $121(13.9)$ & $435(49.1)$ & $256(28.9)$ & $195(22.0)$ \\
\hline \multicolumn{9}{|l|}{ Household income } \\
\hline$<\$ 40,000 /$ year & $133(9.9)$ & $1212(90.1)$ & $576(43.0)$ & $543(40.5)$ & $222(16.6)$ & $599(44.5)$ & $396(29.4)$ & $351(26.1)$ \\
\hline$\geq \$ 40,000 /$ year & $11(2.0)$ & $542(98.0)$ & $369(66.5)$ & $150(27.0)$ & $36(6.5)$ & $301(54.0)$ & $158(28.4)$ & 98 (17.6) \\
\hline \multicolumn{9}{|l|}{ Marital status } \\
\hline $\begin{array}{l}\text { Single, separated, } \\
\text { or divorced }\end{array}$ & $117(26.7)$ & $321(73.3)$ & $153(33.7)$ & $186(41.0)$ & $115(25.3)$ & $148(33.0)$ & $159(35.4)$ & $142(31.6)$ \\
\hline Living together & $36(6.8)$ & 491 (93.2) & $237(46.1)$ & $207(40.3)$ & $70(13.6)$ & 247 (46.9) & $135(25.6)$ & $145(27.5)$ \\
\hline Married & $23(1.8)$ & $1248(98.2)$ & $698(55.4)$ & $428(34.0)$ & $133(10.6)$ & $691(54.5)$ & $360(28.4)$ & $218(17.2)$ \\
\hline \multicolumn{9}{|c|}{$\begin{array}{l}\text { Primary source of payment } \\
\text { for prenatal care }\end{array}$} \\
\hline $\begin{array}{l}\text { Private insurance or } \\
\text { self pay }\end{array}$ & $48(5.2)$ & $884(94.9)$ & $554(59.6)$ & 287 (30.9) & $88(9.5)$ & 469 (49.9) & $273(29.0)$ & $198(21.1)$ \\
\hline $\begin{array}{l}\text { Other insurance or no } \\
\text { insurance }\end{array}$ & $130(9.9)$ & $1179(90.1)$ & $535(41.0)$ & $536(41.1)$ & 234 (17.9) & 618 (47.2) & $384(29.3)$ & $308(23.5)$ \\
\hline
\end{tabular}

${ }^{a}$ Includes Chinese, Japanese, Korean, Vietnamese, Cambodian, Thai, Laotian, Filipino, Indian, other Asian

${ }^{\mathrm{b}}$ Includes Native American, Eskimo, Aleut, Hawaiian, Guamanian, Samoan, Pacific Islanders, and others

${ }^{\mathrm{c}}$ Includes Medicaid, Medicare, Worker's compensation, Title V, No charge, Medically indigent, Medi-Cal CPS Program, no prenatal care, and other government and non-government programs

with higher household incomes, and those who were married. Support levels for foreign-born Latinas remained consistently high across levels of individual demographic factors, except for mothers who were single, separated, or divorced, and those less than 20 years old. For US-born Latinas, support levels varied by demographic factors, with maternal education, household income, marital status, and prenatal care payment source being particularly influential factors.
High levels of chronic stress increased the odds of preterm birth by approximately 50\% (crude odds ratio [cOR] 1.52 [95\%CI 1.19, 1.96]; Table 4), and both measures of pregnancy anxiety were associated with increased preterm birth risk. Contrary to this, stressful life events appeared to be slightly protective (two to six life events adjusted odds ratio [aOR] 0.78 [95\% CI 0.62, 0.99]). Individual life event items showed no associations with preterm birth, but only a few major life events were assessed. 
Table 3 Distributions of paternal support, chronic stress (Perceived Stress Scale), and life event stress by major demographic characteristics among US-born and foreign-born Latinas

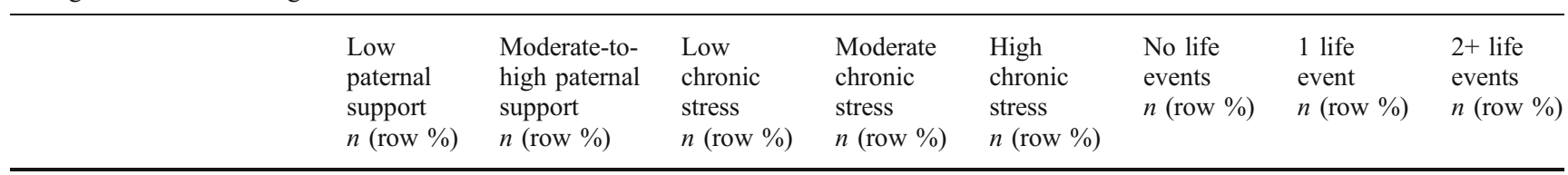

US-born Latinas

Maternal age (years)

$$
\begin{aligned}
& <20 \\
& 20-24 \\
& 25-29 \\
& 30-34 \\
& 35+ \\
& \text { Maternal } \\
& \leq 8 \\
& 9-11 \\
& 12 \\
& 13-15 \\
& 16+ \\
& \text { Parity } \\
& \geq 1
\end{aligned}
$$

Maternal education (years)$$
0
$$

Household income

$<\$ 40,000 /$ year
$\geq \$ 40,000 /$ year
Marital status
Single, separated,
or divorced
Living together
Married

Primary source of payment for prenatal care

Private insurance or self pay

Other insurance or no insurance ${ }^{\mathrm{a}}$

Foreign-born Latinas

Maternal age (years)

$<20$
$20-24$
$25-29$
$30-34$
$35+$

Maternal education (years)

$\leq 8$
$9-11$
12
$13-15$
$16^{+}$
Parity
$\geq 1$
0
Household income
$<\$ 40,000 /$ year
$\geq \$ 40,000 /$ year

$\begin{array}{lrrrrrrr}13(12.3) & 93(87.7) & 38(36.9) & 43(41.8) & 22(21.4) & 52(49.5) & 28(26.7) & 25(23.8) \\ 11(4.6) & 228(95.4) & 93(39.4) & 105(44.5) & 38(16.1) & 136(56.7) & 59(24.6) & 45(18.8) \\ 14(4.7) & 286(95.3) & 129(43.6) & 114(38.5) & 53(17.9) & 156(52.4) & 86(28.9) & 56(18.8) \\ 12(4.4) & 260(95.6) & 135(49.5) & 110(40.3) & 28(10.3) & 147(53.5) & 77(28.0) & 51(18.6) \\ 17(10.8) & 140(89.2) & 73(47.4) & 64(41.6) & 17(11.0) & 86(54.4) & 43(27.2) & 29(18.4) \\ & & & & & & \\ 14(4.9) & 271(95.1) & 107(38.1) & 132(47.0) & 42(15.0) & 179(62.2) & 66(22.9) & 43(14.9) \\ 23(7.3) & 294(92.7) & 135(42.9) & 132(41.9) & 48(15.2) & 180(56.3) & 85(26.6) & 55(17.2) \\ 20(6.7) & 280(93.3) & 135(45.2) & 119(39.8) & 45(15.1) & 136(45.6) & 93(31.2) & 69(23.2) \\ 4(4.3) & 90(95.7) & 47(51.1) & 29(31.5) & 16(17.4) & 44(46.8) & 26(27.7) & 24(25.5) \\ 5(7.9) & 58(92.1) & 36(58.1) & 21(33.9) & 5(8.1) & 32(51.6) & 15(24.2) & 15(24.2) \\ & & & & & & \\ 44(5.9) & 701(94.1) & 313(42.1) & 322(43.3) & 108(14.5) & 397(53.1) & 214(28.6) & 137(18.3) \\ 23(7.0) & 306(93.0) & 155(48.6) & 114(35.7) & 50(15.7) & 180(54.9) & 79(24.1) & 69(21.0) \\ 52(6.7) & 730(93.4) & 341(43.9) & 320(41.2) & 116(14.9) & 404(51.5) & 219(27.9) & 161(20.5) \\ 1(1.3) & 76(98.7) & 44(57.1) & 26(33.8) & 7(9.1) & 43(55.8) & 22(28.6) & 12(15.6)\end{array}$

$\begin{array}{rrrrrrrr}15(17.4) & 71(82.6) & 43(47.8) & 31(34.4) & 16(17.8) & 38(36.9) & 29(32.6) & 21(23.6) \\ 22(14.7) & 128(85.3) & 55(37.4) & 66(44.9) & 26(17.7) & 93(39.4) & 52(35.1) & 50(33.8) \\ 17(13.2) & 112(86.8) & 62(48.1) & 36(27.9) & 31(24.0) & 129(43.6) & 42(32.8) & 41(32.0) \\ 7(11.9) & 52(88.1) & 32(53.3) & 20(33.3) & 8(13.3) & 135(49.5) & 13(22.0) & 17(28.8) \\ 2(12.5) & 14(87.5) & 8(53.3) & 4(26.7) & 3(20.0) & 73(47.4) & 6(40.0) & 1(6.7) \\ 2(33.3) & 4(66.7) & 4(57.1) & 2(28.6) & 1(14.3) & 107(38.1) & 2(28.6) & 2(28.6) \\ 21(18.8) & 91(81.3) & 37(33.0) & 48(42.9) & 27(24.1) & 135(42.9) & 44(39.6) & 25(22.5) \\ 27(14.4) & 160(85.6) & 79(42.3) & 74(39.6) & 34(18.2) & 135(45.2) & 58(31.5) & 62(33.7) \\ 10(10.6) & 84(89.4) & 55(59.1) & 22(23.7) & 16(17.2) & 47(51.1) & 29(30.5) & 30(31.6) \\ 3(7.9) & 35(92.1) & 24(61.5) & 9(23.1) & 6(15.4) & 36(58.1) & 7(18.0) & 10(25.6) \\ 37(14.3) & 221(85.7) & 108(42.4) & 94(36.9) & 53(20.8) & 313(42.1) & 81(32.3) & 78(31.1) \\ 26(14.3) & 156(85.7) & 92(49.5) & 63(33.9) & 31(16.7) & 155(48.6) & 61(32.5) & 52(27.7) \\ 45(15.2) & 251(84.8) & 118(39.9) & 116(39.2) & 62(21.0) & 341(43.9) & 94(31.8) & 103(34.8) \\ 2(2.6) & 76(97.4) & 56(71.8) & 16(20.5) & 6(7.7) & 44(57.1) & 18(23.4) & 17(22.1) \\ 44(33.1) & 89(66.9) & 45(33.1) & 55(40.4) & 36(26.5) & 50(28.7) & 51(38.4) & 40(30.1) \\ 15(12.5) & 105(87.5) & 56(47.1) & 46(38.7) & 17(14.3) & 151(46.8) & 36(30.5) & 44(37.3) \\ 4(2.2) & 182(97.9) & 99(53.5) & 55(29.7) & 31(16.8) & 266(47.6) & 54(28.9) & 46(24.6) \\ 18(9.2) & 177(90.8) & 104(53.6) & 65(33.5) & 25(12.9) & 103(52.0) & 54(27.8) & 57(29.4) \\ 45(18.4) & 200(81.6) & 96(38.9) & 92(37.3) & 59(23.9) & 365(42.3) & 88(35.9) & 73(29.8)\end{array}$


Table 3 (continued)

\begin{tabular}{|c|c|c|c|c|c|c|c|c|}
\hline & $\begin{array}{l}\text { Low } \\
\text { paternal } \\
\text { support } \\
n \text { (row \%) }\end{array}$ & $\begin{array}{l}\text { Moderate-to- } \\
\text { high paternal } \\
\text { support } \\
n(\text { row \%) }\end{array}$ & $\begin{array}{l}\text { Low } \\
\text { chronic } \\
\text { stress } \\
n(\text { row \%) }\end{array}$ & $\begin{array}{l}\text { Moderate } \\
\text { chronic } \\
\text { stress } \\
n(\text { row \%) }\end{array}$ & $\begin{array}{l}\text { High } \\
\text { chronic } \\
\text { stress } \\
n \text { (row \%) }\end{array}$ & $\begin{array}{l}\text { No life } \\
\text { events } \\
n \text { (row \%) }\end{array}$ & $\begin{array}{l}1 \text { life } \\
\text { event } \\
n \text { (row \%) }\end{array}$ & $\begin{array}{l}2+\text { life } \\
\text { events } \\
n \text { (row \%) }\end{array}$ \\
\hline \multicolumn{9}{|l|}{ Marital status } \\
\hline $\begin{array}{l}\text { Single, separated, } \\
\text { or divorced }\end{array}$ & $37(22.2)$ & $130(77.8)$ & $50(28.7)$ & $80(46.0)$ & $44(25.3)$ & $69(40.6)$ & $52(30.6)$ & $49(28.8)$ \\
\hline Living together & $17(5.1)$ & $318(94.9)$ & $151(46.8)$ & $134(41.5)$ & $38(11.8)$ & $184(54.8)$ & $80(23.8)$ & $72(21.4)$ \\
\hline Married & $12(2.1)$ & $556(97.9)$ & $266(47.6)$ & $220(39.4)$ & $73(13.1)$ & $321(56.9)$ & $159(28.2)$ & $84(14.9)$ \\
\hline \multicolumn{9}{|c|}{$\begin{array}{l}\text { Primary source of payment } \\
\text { for prenatal care }\end{array}$} \\
\hline $\begin{array}{l}\text { Private insurance or } \\
\text { self pay }\end{array}$ & $10(5.0)$ & $192(95.1)$ & $103(52.0)$ & $73(36.9)$ & $22(11.1)$ & $107(53.0)$ & $60(29.7)$ & $35(17.3)$ \\
\hline $\begin{array}{l}\text { Other insurance or } \\
\text { no insurance }\end{array}$ & $57(6.6)$ & $812(93.4)$ & $365(42.3)$ & 361 (41.9) & $136(15.8)$ & 469 (53.9) & $232(26.6)$ & $170(19.5)$ \\
\hline
\end{tabular}

${ }^{a}$ Includes Medicaid, Medicare, Worker's compensation, Title V, No charge, Medically indigent, Medi-Cal CPS Program, no prenatal care, and other government and non-government programs

Effect sizes of the various stress measures were similar comparing US- and foreign-born Latinas, except US-born Latinas appeared to have stronger associations between high chronic stress and preterm birth. In adjusted models, high chronic stress was associated with an $83 \%$ increased odds of preterm birth $(95 \%$ CI $1.08,3.11)$ among US-born Latinas, compared to a small and statistically not significant $26 \%$ increased odds among foreign-born Latinas (95\% CI $0.89,1.87$; Table 4$)$, with a $p$ for interaction $=0.17$ between birthplace and high chronic stress. There appeared to be no difference in the effect of support on foreign-born Latinas (aOR 0.97 [95\%CI 0.57, 1.64]) and US-born Latinas (aOR 0.80 [95\%CI $0.45,1.44] ; p$ for interaction $=0.23$ between birthplace and support).

Odds of preterm birth decreased with better support in both crude (cOR 0.67 [95\%CI 0.50, 0.91]) and adjusted models (aOR 0.73 [95\% CI 0.52, 1.01]). To test whether support modified the stress-outcome response, we stratified models of chronic stress by support, adjusting for age, race/ ethnicity, and marital status. Paternal support appeared to mitigate the effects of chronic stress on preterm birth, although confidence intervals were wide. Among women lacking support, those experiencing moderate-to-high levels of chronic stress had higher odds of preterm birth (aOR 2.15 [95\% CI 0.92, 5.03]) compared to those with low stress. In contrast, chronic stress did not increase the risk of preterm birth within the group of women with moderate-tohigh support (aOR 1.13 [95\%CI 0.94, 1.35]). Using interaction terms in our adjusted regression models with a main effect for chronic stress but no main effect for support resulted in a $p$ value of 0.14 for the interaction term. The odds ratios for preterm birth for the other stress measures were similar across the two support strata.

\section{Discussion}

Our study is the first to provide information on paternal support and several types of stress in a large-scale epidemiologic study of adverse birth outcomes, and may be especially informative for studying US-born and immigrant Latina populations in the US. We found low paternal support and chronic stress during pregnancy to be potential risk factors for preterm birth, while women with moderate-to-high levels of support had better outcomes than those with low support. While adjusting for chronic stress and pregnancy anxiety measures moved the effect estimates for paternal support toward the null, the adjusted odds ratios still suggest a protective effect of partner support on preterm birth. Stressful life events were not related to preterm birth risk in our data.

Our study replicated findings from our previous studies of preterm birth using the same PSS and similar measures of pregnancy anxiety (Committee on Understanding Premature Birth and Assuring Healthy Outcomes 2007; Lobel et al. 1992; Rini et al. 1999). A recent North Carolina study also found an increased risk of preterm birth with increased pregnancy anxiety and depression, but did not measure chronic stress (Dole et al., 2003). Although measures of chronic stress and pregnancy anxiety differed somewhat across studies, published studies are suggestive of a positive association with preterm birth similar to ours (Borders et al. 2007).

Previous studies of life event stress have reported both positive or null associations with preterm birth (Committee on Understanding Premature Birth and Assuring Healthy Outcomes 2007; Dunkel-Schetter and Glynn 2010; Hoffman and Hatch 1996; Khashan et al. 2009; Lu and Chen 2004; 


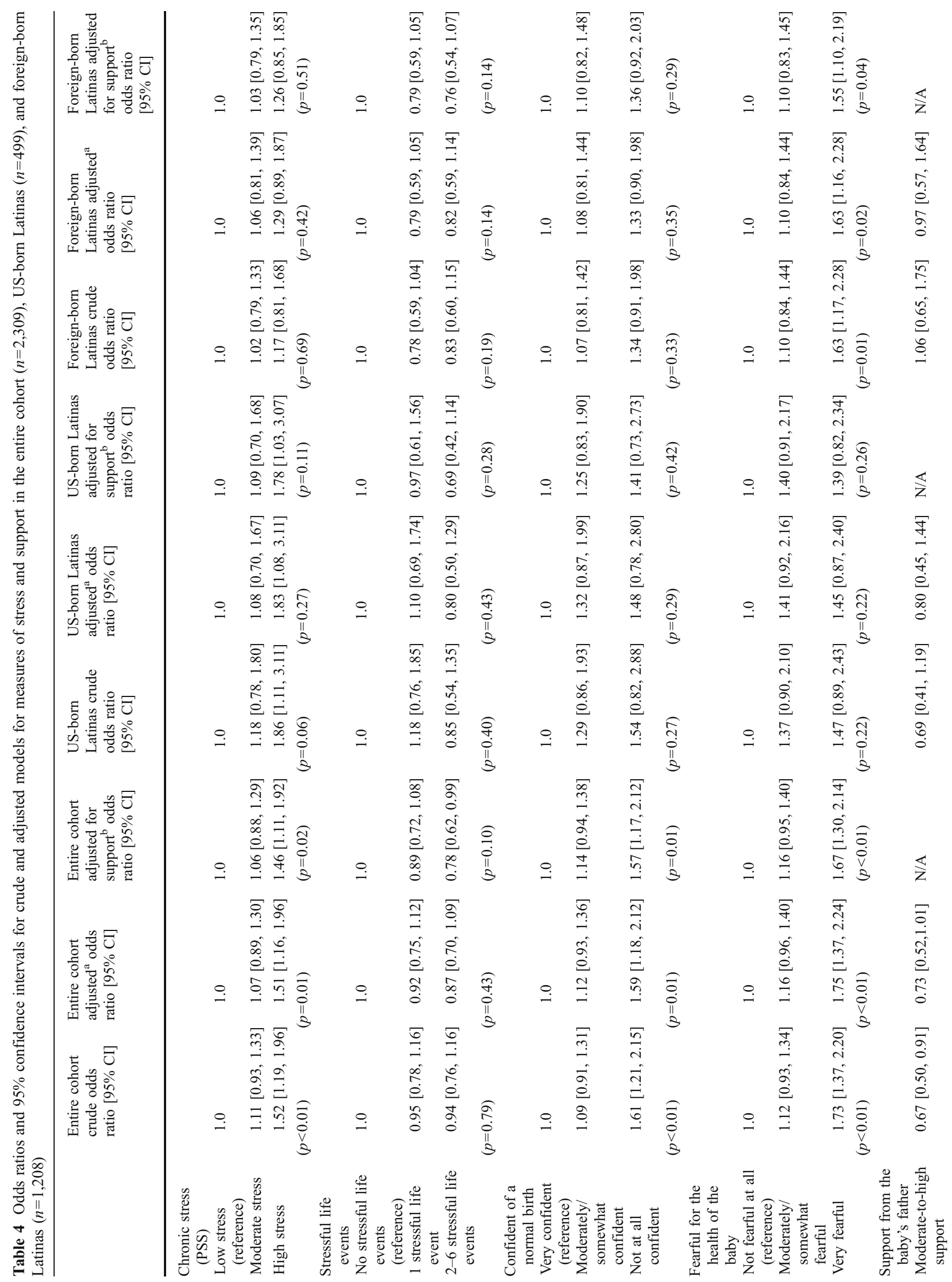




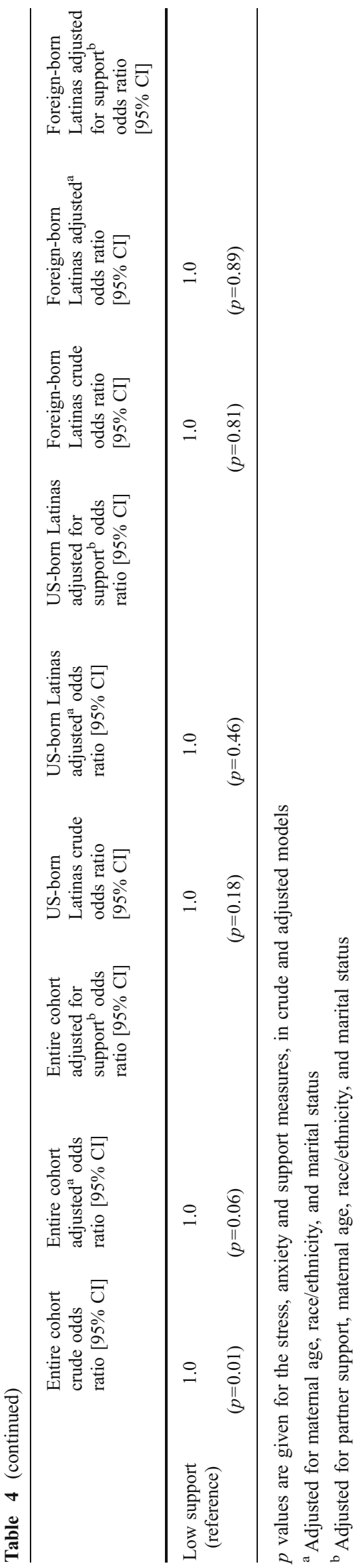

Nordentoft et al. 1996; Obel et al. 2003). In particular, two large-scale studies reported risk increases when life events were weighted according to their perceived impact. First, a prospective study in North Carolina (Dole et al. 2003) reported increased risk with life events with negative impact in primiparae recruited from prenatal clinics at or before 24-30 weeks of gestation. Similarly, a Danish study of women from a hospital-based antenatal care program found that life events occurring between 16 and 30 weeks of gestation and perceived as highly stressful increased preterm birth risk (Hedegaard et al. 1996). Our null findings are not necessarily surprising. We believe to have selected six life events of most relevance to our participant sample and region. However, it seems likely that we missed identifying some of the most upsetting events because we did not ask about all events of importance during pregnancy, could not weigh each item by its perceived impact, and could not assess which events occurred during the most critical time windows for preterm birth. We would have expected more life events to be recalled by women with adverse birth outcomes in an attempt to explain these outcomes, such that recall bias would have produced biased positive associations and not the reverse as observed here. On the other hand, our study may not have been able to recruit women who experienced the most severe life events (i.e., severe events followed by an adverse pregnancy outcome may have caused some response bias). Nevertheless, our results agree with those previous studies, some prospective, that did not identify an increased risk for life event stress (Hoffman and Hatch 1996; Lobel et al. 1992; Nordentoft et al. 1996; Pagel et al. 1990).

Previous birth outcomes studies of support have generally reported no associations for preterm birth. The Danish (Hedegaard et al. 1996) study reported that social support did not buffer the detrimental effects of life event stress on preterm birth. The New Zealand study (Pryor et al. 2003) using the Family Support Scale (Dunst et al. 1984) found no effect of formal social support (e.g., school- or communitybased programs, and professional services) on small for gestational age (SGA) risk, but found that informal social support (e.g., friends, family and partners) was protective for SGA; however, the association disappeared after adjustment for race/ethnicity. A recent Cochrane review of support intervention studies found no effect on gestational age (Hodnett and Fredericks 2003). In contrast, our results showed a small moderating effect for support from the baby's father, even after adjusting for maternal age, race/ ethnicity, and marital status. A North Carolina study of preterm birth found a small buffering effect for general social support on chronic stress (Dole et al. 2003), yet the same group reported no effect of support in a subsequent publication using a subset of the dataset from the earlier study (Dole et al. 2004). 
There are several important features of our study that differ from previous research, including the type of support we measured and the fact that the majority of our population was Latina. Our study focused only on support from the baby's father, which is suspected to be the most significant source of support in relation to prenatal stress (Rini et al. 2006). However, the relative importance of different forms of support, especially within culturally diverse populations, may be important when interpreting study results and in planning intervention programs.

Studies suggested that non-Latino whites have large support networks of friends, but nevertheless derive emotional and material support primarily from their partners. In contrast, the extended family network is the primary source of support for Latinas (Campos et al. 2008; Sagrestano et al. 1999). Recent immigrant women with fewer family members living nearby may receive more support from their husbands. In our study, more foreign-born Latinas reported receiving moderate-to-high support from the baby's father, compared to white non-Latina mothers, while US-born Latinas reported lower levels of support. Much of this difference is likely due to higher proportions of Whites and foreign-born Latinas being married, which is strongly associated with better birth outcomes and also highly predictive of partner support (Lee et al. 2006). Consistent with other studies in Los Angeles (Zambrana et al. 1997), we found that US-born Latinas reported more stress of all types than foreign-born Latinas, despite foreign-borns having lower household incomes and being less educated. Over $75 \%$ of the US-born Latinas completed our survey in English compared to only $15 \%$ of foreign-born Latinas, suggesting a large difference in degree of acculturation. Thus, it is likely that US-born Latinas may have adopted more American beliefs and values, which may influence how they perceive and internalize support and stress.

Recall bias is a potential limitation in all retrospective case-control studies. At the time of the interview, it is possible that mothers caring for a preterm baby may not accurately recall the degree of stress experienced during pregnancy. For measures of pregnancy anxiety and chronic stress, our estimates may be biased upward due to overreporting of stress with case babies. Furthermore, the mothers' assessment of support may also be biased based on their experiences post-delivery, but the direction of this bias is not easily predicted. Mothers were interviewed no more than 3-6 months after the baby's birth to reduce recall bias, and this might also have made the efforts and stress related to taking care of a baby-whether preterm or term-more similar than if we had interviewed them right after birth. Additionally, the pregnancy anxiety measures are more likely to be subject to case status bias because they ask specifically about the pregnancy and the baby. The chronic stress measures ask more generally about the woman's experiences during pregnancy, and we expect these measures to be less subject to recall bias.

Because study participants were recruited from all registered births based on birth certificate addresses, we were unable to contact a large proportion of the mothers, allowing for potential response bias in our study. We conducted extensive analyses comparing responders, nonresponders, and the overall birth cohort, and found few differences (Ritz et al. 2007). It is plausible, however, that women caring for a preterm baby who experienced high stress were less likely to respond to our survey than those experiencing less stress and caring for a normal weight term baby. Such differential selection would negatively bias the stress measure associations and may explain our null and protective results for life event stress, or attenuate associations observed for chronic stress and pregnancy anxiety. In contrast to many existing birth outcome studies of social support and stress, ours is a truly population-based sample from a large birth cohort rather than a sample of women recruited from prenatal clinics and hospitals. We were able to recruit a large number of Spanish-speaking women ( $n=955$ of the 2,543 EPOS participants), over $95 \%$ of whom were foreign-born. Selection bias is also a potential problem in clinic-based case-control studies when the control group is not representative of the base population. Also, pregnant women recruited from clinics may not be representative of all women giving birth in the population. Therefore, despite the potential for response and recall bias in our study, the use of a population-based birth cohort promises higher generalizability of results to similar source populations.

As with all birth-certificate-based studies, the data quality is a potential limitation, with gestational age information being somewhat problematic (Northam and Knapp 2006). In our survey, we confirmed the date of birth of the child, but did not verify gestational age since we did not have access to medical records. A more accurate measure of gestational age would require ultrasound records from the first trimester, which are unlikely to be available in a large percentage of the women in our study with low socioeconomic status. Therefore, although the outcome assignment may be imperfect, our data may be the best available on a broad population level for a largely recent immigrant population with limited access to health care services.

In conclusion, our results suggest that support from the baby's father may mitigate or alleviate some of the effects of chronic stress on preterm birth. Because our study used a large population-based sample, and measured paternal support and several different types of stress, we were able to evaluate the relative influence of these different forms of stress and anxiety, along with the effect of paternal support. While the retrospective nature of our study may have introduced some bias, at minimum, our data may help to identify the women at highest risk for adverse outcomes, 
and inform intervention programs that address specific types of stress and pregnancy anxiety in immigrant populations. We recommend that future population-based studies cover a broader spectrum of stressors and social support to distinguish between the effects of source and type of support and stress on pregnancy outcomes among mothers from diverse cultures.

Open Access This article is distributed under the terms of the Creative Commons Attribution Noncommercial License which permits any noncommercial use, distribution, and reproduction in any medium, provided the original author(s) and source are credited.

\section{References}

Badr LK, Abdallah B, Mahmoud A (2005) J Obstet Gynecol Neonatal Nurs 34:444-452

Borders AE, Grobman WA, Amsden LB, Holl JL (2007) Obstet Gynecol 109:331-338

Bryce RL, Stanley FJ, Enkin MW (1988) Birth 15:19-23

Campos B, Dunkel Schetter C, Abdou CM, Hobel CJ, Glynn LM, Sandman CA (2008) Cultur Divers Ethnic Minor Psychol 14:155-162

Cohen S, Kamarck T, Mermelstein R (1983) J Health Soc Behav 24:385-396

Collins NL, Dunkel-Schetter C, Lobel M, Scrimshaw SC (1993) J Pers Soc Psychol 65:1243-1258

Committee on Understanding Premature Birth and Assuring Healthy Outcomes (2007) Preterm birth: causes, consequences, and prevention. National Academies Press, Washington, DC

Copper RL, Goldenberg RL, Das A, Elder N, Swain M, Norman G, Ramsey R, Cotroneo P, Collins BA, Johnson F, Jones P, Meier AM (1996) Am J Obstet Gynecol 175:1286-1292

Dole N, Savitz DA, Hertz-Picciotto I, Siega-Riz AM, McMahon MJ, Buekens P (2003) Am J Epidemiol 157:14-24

Dole N, Savitz DA, Siega-Riz AM, Hertz-Picciotto I, McMahon MJ, Buekens P (2004) Am J Public Health 94:1358-1365

Dominguez TP, Dunkel-Schetter C, Glynn LM, Hobel C, Sandman CA (2008) Health Psychol 27:194-203

Dudley DJ (1999) Am J Obstet Gynecol 180:S251-S256

Dunkel-Schetter, C and Glynn, L (2010) In Handbook of Stress (Eds, R. Contrada and A. Baum). In press

Dunst CJ, Jenkins V, Trivette CM (1984) J Indiv Fam Comm Wellness $1: 45-52$

Elsenbruch S, Benson S, Rucke M, Rose M, Dudenhausen J, PincusKnackstedt MK, Klapp BF, Arck PC (2007) Hum Reprod 22:869-877

Feldman PJ, Dunkel-Schetter C, Sandman CA, Wadhwa PD (2000) Psychosom Med 62:715-725

Hedegaard M, Henriksen TB, Sabroe S, Secher NJ (1993) BMJ 307:234-239

Hedegaard M, Henriksen TB, Secher NJ, Hatch MC, Sabroe S (1996) Epidemiology 7:339-345

Hobel CJ (2004) Clin Obstet Gynecol 47:856-880, discussion 881-2

Hobel C, Culhane J (2003) J Nutr 133:1709S-1717S

Hobel CJ, Goldstein A, Barrett ES (2008) Clin Obstet Gynecol 51:333-348

Hodnett, ED and Fredericks, S (2003) Cochrane Database Syst Rev, CD000198
Hoffman S, Hatch MC (1996) Paediatr Perinat Epidemiol 10:380-405

Hogue CJ, Hoffman S, Hatch MC (2001) Paediatr Perinat Epidemiol 15(Suppl 2):30-40

Khashan AS, McNamee R, Abel KM, Mortensen PB, Kenny LC, Pedersen MG, Webb RT, Baker PN (2009) Hum Reprod 24:429_ 437

Knackstedt MK, Hamelmann E, Arck PC (2005) Am J Reprod Immunol 54:63-69

Kramer MS, Goulet L, Lydon J, Seguin L, McNamara H, Dassa C, Platt RW, Chen MF, Gauthier H, Genest J, Kahn S, Libman M, Rozen R, Masse A, Miner L, Asselin G, Benjamin A, Klein J, Koren G (2001) Paediatr Perinat Epidemiol 15(Suppl 2):104-123

Lee TY, Miles MS, Holditch-Davis D (2006) J Obstet Gynecol Neonatal Nurs 35:46-55

Lobel M, Dunkel-Schetter C, Scrimshaw SC (1992) Health Psychol 11:32-40

Lu MC, Chen B (2004) Am J Obstet Gynecol 191:691-699

Martin, JA, Hamilton, BE, Sutton, PD, Ventura, SJ, Menacker, F and Kirmeyer, S (2006) National vital statistics reports, 55

McLean M, Smith R (2001) Reproduction 121:493-501

Nordentoft M, Lou HC, Hansen D, Nim J, Pryds O, Rubin P, Hemmingsen R (1996) Am J Public Health 86:347-354

Northam S, Knapp TR (2006) J Obstet Gynecol Neonatal Nurs 35:3-12

Obel C, Hedegaard M, Henriksen TB, Secher NJ, Olsen J (2003) Dev Med Child Neurol 45:802-806

Paarlberg KM, Vingerhoets AJ, Passchier J, Dekker GA, Van Geijn HP (1995) J Psychosom Res 39:563-595

Pagel MD, Smilkstein G, Regen H, Montano D (1990) Soc Sci Med 30:597-604

Pryor JE, Thompson JM, Robinson E, Clark PM, Becroft DM, Pattison NS, Galvish N, Wild CJ, Mitchell EA (2003) Acta Paediatr 92:62-64

Raghunathan, T, Solenberger, P and Van Hoewyk, J (2002) University of Michigan

Rini CK, Dunkel-Schetter C, Wadhwa PD, Sandman CA (1999) Health Psychol 18:333-345

Rini CM, Dunkel Schetter C, Hobel CJ, Glynn LM, Sandman CA (2006) Personal Relationships 13:207-222

Ritz B, Wilhelm M, Hoggatt KJ, Ghosh JK (2007) Am J Epidemiol 166:1045-1052

Roesch SC, Dunkel-Schetter C, Woo G, Hobel C (2004) Anxiety, Stress, and Coping 17:87-102

Rondo PH, Ferreira RF, Nogueira F, Ribeiro MC, Lobert H, Artes R (2003) Eur J Clin Nutr 57:266-272

Rothberg AD, Lits B (1991) Am J Obstet Gynecol 165:403-407

Ruiz RJ, Fullerton J, Brown CE, Dudley DJ (2002) Biol Res Nurs 4:54-64

Sagrestano LM, Feldman P, Rini CK, Woo G, Dunkel-Schetter C (1999) Am J Community Psychol 27:869-898

Sandman CA, Glynn L, Schetter CD, Wadhwa P, Garite T, ChiczDeMet A, Hobel C (2006) Peptides 27:1457-1463

Turner RJ, Grindstaff CF, Phillips N (1990) J Health Soc Behav 31:43-57

Villar J, Farnot U, Barros F, Victora C, Langer A, Belizan JM (1992) N Engl J Med 327:1266-1271

Wadhwa PD, Sandman CA, Porto M, Dunkel-Schetter C, Garite TJ (1993) Am J Obstet Gynecol 169:858-865

Wadhwa PD, Culhane JF, Rauh V, Barve SS, Hogan V, Sandman CA, Hobel CJ, Chicz-DeMet A, Dunkel-Schetter C, Garite TJ, Glynn L (2001) Paediatr Perinat Epidemiol 15(Suppl 2):17-29

Wadhwa PD, Garite TJ, Porto M, Glynn L, Chicz-DeMet A, DunkelSchetter C, Sandman CA (2004) Am J Obstet Gynecol 191:1063-1069

Weinstock M (2005) Brain Behav Immun 19:296-308

Zambrana RE, Scrimshaw SC, Collins N, Dunkel-Schetter C (1997) Am J Public Health 87:1022-1026 